\title{
Magnetization transfer effects on the efficiency of flow-driven adiabatic fast passage inversion of arterial blood
}

\author{
Luis Hernandez-Garcia, ${ }^{1 *}$ David P. Lewis, ${ }^{2,3}$ Bradford Moffat $^{4}$ and Craig A. Branch ${ }^{2,3}$ \\ ${ }^{1}$ FMRI Laboratory, University of Michigan, Ann Arbor, MI, USA \\ ${ }^{2}$ Center for Advanced Brain Imaging \& Division of Medical Physics, The Nathan S. Kline Institute for Psychiatric Research, Orangeburg, NY, USA \\ ${ }^{3}$ Department of Radiology and Neuroscience, Albert Einstein College of Medicine, Bronx, NY, USA \\ ${ }^{4}$ Department of Radiology, University of Michigan, Ann Arbor, MI, USA
}

Received: 24 June 2006; Revised 3 November 2006; Accepted 28 November 2006

\begin{abstract}
Continuous arterial spin labeling experiments typically use flow-driven adiabatic fast passage inversion of the arterial blood water protons. In this article, we measure the effect of magnetization transfer in blood and how it affects the inversion label. We use modified Bloch equations to model flow-driven adiabatic inversion in the presence of magnetization transfer in blood flowing at velocities from 1 to $30 \mathrm{~cm} / \mathrm{s}$ in order to explain our findings. Magnetization transfer results in a reduction of the inversion efficiency at the inversion plane of up to $3.65 \%$ in the range of velocities examined, as well as faster relaxation of the arterial label in continuous labeling experiments. The two effects combined can result in inversion efficiency reduction of up to $8.91 \%$ in the simulated range of velocities. These effects are strongly dependent on the velocity of the flowing blood, with $10 \mathrm{~cm} / \mathrm{s}$ yielding the largest loss in efficiency due to magnetization transfer effects. Flowing blood phantom experiments confirmed faster relaxation of the inversion label than that predicted by $T_{1}$ decay alone. Copyright (C) 2007 John Wiley \& Sons, Ltd.
\end{abstract}

KEYWORDS: adiabatic inversion; blood flow; magnetization transfer; CBF; arterial spin labeling; MRI

\section{INTRODUCTION}

Cerebral perfusion measurements using arterial spin labeling (ASL) techniques are typically carried out by inverting arterial water protons upstream of the tissue of interest, and measuring the MR signal change as the inverted protons diffuse into the tissue. Either a bolus (pulsed ASL) (1-4) or a steady-state tag (continuous ASL) (5-8) can be employed, by inverting or saturating arterial water spins. In the case of continuous ASL, the inversion of inflowing arterial water protons is usually accomplished through flow-driven adiabatic inversion. In short, a long (3-4 s) radio frequency (RF) pulse is applied in the presence of a magnetic field gradient in the direction of the flow (typically the $z$-axis). Thus, as the arterial water spins flow through the gradient, their Larmor frequency changes with their position at a rate determined by their speed and the strength of the gradient. From the point of view of the moving spins, it appears as if the RF pulse sweeps over a range of frequencies relative

*Correspondence to: L. Hernandez-Garcia, University of Michigan FMRI Laboratory, 2360 Bonisteel Ave., Ann Arbor, MI 28109-2108, USA.

E-mail: hernan@umich.edu

Contract/grant sponsor: National Institute of Biomedical Imaging and Bioengineering; contract/grant number: 1 R01 EB004346-01A1.

Abbreviations used: ASL, arterial spin labeling; RF, radio frequency. to the resonance frequency, although the applied RF pulse has constant frequency. The gradient and the RF frequency are chosen such that the moving spins are on resonance only at the center of the inversion slice. If the frequency sweep is significantly slower than the precession caused by the $H_{1}$ field, but faster than $T_{2}$ decay, the spin ensemble tracks the net magnetic field from the positive $z$-axis to the negative $z$-axis and the spins become inverted. This phenomenon is commonly referred to as "spin locking". The conditions for spin locking are summarized by:

$$
1 / T_{2} \ll G_{z} V_{z} / H_{1} \ll \gamma H_{1}
$$

where $G_{\mathrm{z}}$ is the magnetic field gradient strength, $V_{\mathrm{z}}$ is the velocity of the spin perpendicular to the labeling plane, $\gamma$ is the gyromagnetic ratio, and $H_{1}$ is the field strength of the inversion pulse (9). The degree of inversion achieved by the off-resonance RF radiation is described by:

$$
\alpha=\frac{M_{a}^{0}-M_{a}^{i n v}}{2 M_{a}^{0}}
$$

where, $M_{a}^{0}$ is the relaxed longitudinal magnetization of arterial spins, and $M_{a}^{i n v}$ is the longitudinal magnetization of arterial spins after they move through the inversion plane. 
Whereas long $T_{2}$ spins (the free protons in arterial water) experience the adiabatic inversion at the inversion plane, those that have short $T_{2}$ (protons that are bound to macromolecules such as albumin or cholesterol) do not meet the adiabatic condition and are saturated over the time period the pulse is applied. The bound protons absorb the RF during the entire frequency sweep (i.e. the trajectory over the magnetic field gradient) because of their broad absorption spectrum. Chemical exchange between the two proton pools potentially results in magnetization transfer and partial loss of the degree of inversion.

Blood is typically thought to have little or no magnetization transfer but, in a study of Time-of-Flight angiography, Doussett et al. (10) and Pike et al. (11) observed that saturation transfer occurs not only in tissue, but also in flowing blood spins when off-resonance radiation is applied. They also observed that this saturation transfer is heavily dependent on flow velocity. This effect was also reported by us in a preliminary experiment for adiabatic inversions in continuous ASL $(12,13)$. Magnetization transfer in blood arising from background suppression pulses has recently been found to affect inversion efficiency in pulsed ASL by Garcia et al. (14). In this article, we will explore the question of how magnetization transfer affects the inversion efficiency in continuous arterial spin labeling experiments.

Formulations for optimization of the continuous arterial spin labeling experiment have been presented previously for both the single (15) and double (16) coil experiments, providing general guidelines for choice of the adiabatic slice selection gradient, RF transmitter power and RF offset frequency for optimizing the inversion process, and minimizing the RF power required, and thus specific absorption rate $(17,18)$. Using numerical solutions to the modified Bloch equations, Maccotta et al. (19) indicated that measured adiabatic efficiencies in humans approach simulated levels, provided that one accounts for $T_{1}$ relaxation occurring during vascular transit periods. Whereas simulated efficiencies can approach $96 \%$ for proper choices of gradient and RF parameters, measured efficiencies generally approach $75-90 \%$ before correction for $T_{1}$ relaxation $(8,15,19)$. However, the simulation studies did not include the effect of magnetization transfer within the blood. In this report, we model the flow-driven adiabatic inversion pulse including magnetization transfer effects and compare our findings with blood phantom measurements in flowing and stationary blood.

\section{METHODS}

\section{Simulations}

We consider blood as a spin system composed of two general pools of protons: free and bound. The bound protons are those that are trapped in, or exchanged with, the macromolecules present in blood, such as albumin, cholesterol, hemoglobin, etc. In a single-coil continuous ASL experiment, the tagged spins continue to be irradiated after the inversion, as they travel from the inversion plane toward the brain. The frequency offset (relative to resonance) of the RF experienced by the moving spins increases as the spins travel away from the inversion plane because of the presence of the slice selection gradient.

The classical Bloch equations can be used to describe the net magnetization of a spin system in the rotating frame of reference and in the presence of off-resonance RF radiation of intensity $H_{1}$ applied along the $y$-axis, at frequency offset $\left(\omega-\omega_{0}\right)$. For moving arterial spins, the frequency offset of the $H_{1}$ pulse evolves according to:

$$
\omega-\omega_{0}=\gamma G_{z} V_{z} t
$$

The frequency offset sweeps from $-\omega_{1}$ to $\omega_{2}$ passing through zero at the center of the inversion slice. Assuming that these spins have a constant velocity component in the direction of the slice-select magnetic field gradient, the Bloch equations can be modified as

$$
\begin{aligned}
& \frac{d M_{z}(t)}{d t}=-\frac{M_{z}(t)-M(0)}{T_{1}}-\gamma H_{1} M_{y}(t) \\
& \frac{d M_{x}(t)}{d t}=-\frac{M_{x}(t)}{T_{2}}+G_{z} V_{z} t M_{y}(t) \\
& \frac{d M_{y}(t)}{d t}=-\frac{M_{y}(t)}{T_{2}}-G_{z} V_{z} t M_{x}(t)+\gamma H_{1} M_{z}(t)
\end{aligned}
$$

Magnetization transfer effects can be taken into consideration by modifying the Bloch equations to include cross-relaxation and chemical exchange $(20,21)$ between free and bound protons within the arterial blood. Thus, the Bloch equations for two separate pools of moving protons, free (a) and bound (b), including terms for chemical exchange between the two pools are:

Pool a:

$$
\begin{aligned}
& \frac{d M_{z}^{a}(t)}{d t}=-\frac{M_{z}^{a}(t)-M^{a}(0)}{T_{1 a}}-\gamma H_{1} M_{y}^{a}(t)+k_{b} M_{z}^{b}(t)-k_{a} M_{z}^{a}(t) \\
& \frac{d M_{x}^{a}(t)}{d t}=-\frac{M_{x}^{a}(t)}{T_{2 a}}+G_{z} V_{z} t M_{y}^{a}(t) \\
& \frac{d M_{y}^{a}(t)}{d t}=-\frac{M_{y}^{a}(t)}{T_{2 a}}-G_{z} V_{z} t M_{x}^{a}(t)+\gamma H_{1} M_{z}^{a}(t)
\end{aligned}
$$

Pool b:

$$
\begin{aligned}
& \frac{d M_{z}^{b}(t)}{d t}=-\frac{M_{z}^{b}(t)-M^{b}(0)}{T_{1 b}}-\gamma H_{1} M_{y}^{b}(t)+k_{a} M_{z}^{a}(t)-k_{b} M_{z}^{b}(t) \\
& \frac{d M_{x}^{b}(t)}{d t}=-\frac{M_{x}^{b}(t)}{T_{2 b}}+G_{z} V_{z} t M_{y}^{b}(t) \\
& \frac{d M_{y}^{b}(t)}{d t}=-\frac{M_{y}^{b}(t)}{T_{2 b}}-G_{z} V_{z} t M_{x}^{b}(t)+\gamma H_{1} M_{z}^{b}(t)
\end{aligned}
$$


Table 1. Definitions of variables and their values in the model

\begin{tabular}{llc}
\hline Symbol & Definition & Value in model \\
\hline$H_{1}$ & RF magnetic field strength & $100 \mathrm{mG}$ \\
$M_{\mathrm{x}, \mathrm{y}, \mathrm{z}}(\mathrm{t})$ & Three-space components of the magnetization vector & $42.58 \mathrm{MHz} / \mathrm{T}$ \\
$\gamma$ & as a function of time & $0.062,280 \times 10^{-6} \mathrm{~s}$ \\
$T_{2 \mathrm{a}, \mathrm{b}}$ & Gyromagnetic ratio & $1.85,0.5 \mathrm{~s}$ \\
$T_{1 \mathrm{a}, \mathrm{b}}$ & Transverse relaxation rate for each individual pool & $1.36 \mathrm{~s}$ \\
$T_{1}$ sat & Longitudinal relaxation rate for each individual pool & $35 \mathrm{~s} \mathrm{~s}^{-1}$ \\
$K_{\mathrm{a}}$ & $T_{1}$ relaxation rate in the presence of saturation transfer & $0.98 \mathrm{~s}$ \\
$K_{\mathrm{b}}$ & Chemical exchange rate from pool a to pool b & 0.028 \\
$F$ & Chemical exchange rate from pool b to pool a & +1.0 \\
$M_{\mathrm{z}}(0)$ & Fraction of protons in pool b relative to pool a & $780 \mathrm{mG} / \mathrm{cm}$ \\
$G_{\mathrm{z}}$ & Initial condition: all spins fully relaxed & $15 \mathrm{~cm} / \mathrm{s}$ \\
$V_{\mathrm{z}}$ & Magnetic field gradient in the direction of flow & $5000 \mathrm{~Hz}$ \\
$\omega-\omega_{0}$ & z-component of flow velocity & \\
\hline
\end{tabular}

where $k_{\mathrm{a}}$ and $k_{\mathrm{b}}$ are the forward and backward exchange rates between the two pools of protons. If the fraction of bound protons to free protons is given by $f$, the NMR signal is obtained from sampling $M_{\mathrm{z}}^{\mathrm{a}}+f M_{\mathrm{z}}^{\mathrm{b}}$. To investigate the relaxation of the labeled spins under these conditions as they move toward the brain, we numerically solved the above system of differential equations numerically using a Runge-Kutta method (Matlab; The Mathworks, Nattick, MA, USA) from $t=-1$ to $1 \mathrm{~s}$, letting $t=0$ be the time when the spins cross the inversion plane. The RF radiation was kept constant during the whole time span of the simulation. The initial condition for both pools of spins was the fully relaxed longitudinal state. Unless otherwise specified, the parameters used in the simulations are given in Table 1. We simulated the inversion process under magnetization transfer conditions at several velocities from 1 to $30 \mathrm{~cm} / \mathrm{s}$. We also simulated the inversion without magnetization transfer at $20 \mathrm{~cm} / \mathrm{s}$ by letting the exchange rate constants, $k_{\mathrm{a}}$ and $k_{\mathrm{b}}$, equal zero and hence letting the signal be determined by the behavior of the free pool of protons.

\section{Phantom studies: stationary spins}

Venous human blood was extracted from two healthy men and preserved with heparin to avoid coagulation. The phantom was monitored visually for possible coagulation or sedimentation, as the experiments lasted for several hours from beginning to end. All measurements were carried out at room temperature $\left(22^{\circ} \mathrm{C}\right)$.

Decay rates of human blood were measured using a Varian 7 Tesla scanner using a bird cage RF coil (Varian, Palo Alto, CA, USA) as follows. $T_{1}$ was measured using an inversion recovery experiment consisting of a hyperbolic-secant adiabatic inversion pulse followed by a spin-echo acquisition ( $T R=12 \mathrm{~s}, T E=14.5 \mathrm{~ms}$, bandwidth $(\mathrm{BW})=20 \mathrm{kHz}$, number of excitations $(\mathrm{NEX})=2$ ). Inversion times were 50, 100, 200, 500, 1000, 2000, 5000, 8000 , and $10000 \mathrm{~ms}$. Data were fitted by a non-linear least squares algorithm (Levenberg-Marquardt) to the inversion recovery equation including $T R$ effects. $T_{2}$ was measured using a fast spin echo sequence $(T R=8$, echo train length $=32, T E=15 \mathrm{~ms}$ per echo, $\mathrm{NEX}=2$, $\mathrm{BW}=20 \mathrm{kHz}$ ). Data from alternating echoes (to avoid phase errors) were fitted by a non-linear least squares algorithm to the $T_{2}$ exponential decay equation.

The magnetization transfer spectrum of human blood was measured on a 7.0 T magnet (Magnex Scientific, Abingdon, Oxon, UK) with a SMIS spectrometer (Surrey Medical Imaging Systems, Guilford, Surrey, UK). Images were collected using a 16-rod bird cage coil, $8 \mathrm{~cm}$ in diameter. The experiment consisted of a spin echo sequence preceded by a pre-saturation pulse applied for $2 \mathrm{~s}$ at frequencies ranging from 100 to $15000 \mathrm{~Hz}$ off-resonance. The pre-saturation pulse duration and power were determined at $5000 \mathrm{~Hz}$ off-resonance by gradual increases until the MR signal reached equilibrium. The same experiment was carried out on water for comparison purposes. The forward exchange rate between free and bound protons, $k_{\mathrm{a}}$, was calculated by a non-linear least squares fit of the model presented by Henkelman et al. (20). In notation consistent with the present paper, the equation describing the magnetization transfer is:

$$
\frac{M_{z}^{a}\left(\omega_{1}\right)}{M_{0}^{a}}=\frac{R_{1 b} k_{a}+R_{r f b} R_{1 a}+R_{1 b} R_{1 a}+R_{1 a} k_{a} f}{\left(R_{1 a}+R_{f a}+R k_{a}\right)\left(R_{1 b}+R_{r f b}+k_{a} f\right)-k_{a} f k_{a}}
$$

where

$$
\begin{aligned}
R_{r f a} & =\frac{\omega_{1}^{2} T_{2 a}}{1+\left[2 \pi\left(\omega_{1}-\omega_{0}\right) T_{2 a}\right]} \text { and } \\
R_{r f b} & =\frac{\omega_{1}^{2} T_{2 b}}{1+\left[2 \pi\left(\omega_{1}-\omega_{0}\right) T_{2 b}\right]}
\end{aligned}
$$

All parameters in the equation were fixed except for the rate constant of interest. $T_{2}$ of the bound proton pool was assumed to be $0.28 \mathrm{~ms}$, and the fraction of bound to free protons was 0.028, as reported in Stanisz et al. (22). Values for the $T_{1}$ rate of the bound protons were not found 
in the literature. However, one can expect the value to be much smaller than those of the free pool, in which case, the parameter has a very small effect on the simulations. Hence, $T_{1}$ of the bound pool was arbitrarily assumed to be $500 \mathrm{~ms}$.

$T_{1}$ sat of blood was measured by a progressive saturation experiment in which the sample was radiated with off-resonance RF $(5000 \mathrm{~Hz}$ offset) between acquisitions in order to achieve an equilibrium saturation transfer. TR were 200, 500, 1000, 2000, 5000, $10000 \mathrm{~ms}$. Acquisition was performed using a line scan acquisition $(23,24)$. The data were fitted using a least squares fit to the progressive saturation equation.

\section{Phantom studies: flowing spins}

To examine the influence of flow velocity on magnetization transfer in the ASL measurement, we measured the magnetization transfer ratio after an adiabatic fast passage pulse in both flowing tap water and blood. A flow phantom was constructed consisting of $0.25-\mathrm{cm}$ internal diameter Tygon $^{\mathrm{TM}}$ tubing passing through the RF coil in the magnet between two containers. The flow was driven by a ministaltic recirculating pump. Flow velocity was measured by collecting a $T_{1}$-weighted sagittal image [spin echo, $T R / T E=300 / 21 \mathrm{~ms}$, field of view $(\mathrm{FOV})=64 \mathrm{~mm}$, $128 \times 128$ ] acquired $1 \mathrm{~ms}$ after a $200 \mathrm{~ms}$ adiabatic fast passage inversion pulse. Velocity was calculated from the peak distance traveled by the inverted spins during the inversion pulse.

The degree of adiabatic inversion as a function of inversion pulse power was examined in both blood and water at different flow velocities ranging from 2 through $24 \mathrm{~cm} / \mathrm{s}$. After measurement of flow velocity as described, a set of transverse GE images $(T R / T E=300 / 9 \mathrm{~ms}$, $\mathrm{FOV}=32 \mathrm{~mm}, \quad 128 \times 128)$ was collected $10-20 \mathrm{~mm}$ downstream from an inversion plane of variable $\mathrm{RF}$ power from 0 to $200 \mathrm{mG}$. The effective adiabatic inversion efficiency, $\alpha^{\prime}$, was computed using eqn (2) from the magnitude and phase of the signal within a circular region of interest of area $0.6 \mathrm{~cm}^{2}$ in the center of the flow phantom identified on the transverse images and corresponding to the fastest flowing component of the parabolic flow profile. This radius was chosen to be small enough to contain approximately the water spins moving within $10 \%$ of the peak velocity. After measurement of $\alpha^{\prime}$, the absolute degree of inversion, $\alpha$, was calculated by correcting for $T_{1}$ decay of the inversion. We then measured the maximum inversion efficiency attainable over a range of velocities in blood and identified the relative power required to achieve that level of inversion.

To examine the relaxation of flowing inverted spins in the presence of off-resonance RF, sagittal images of flowing blood and water $(\sim 10 \mathrm{~cm} / \mathrm{s})$ were collected after application of a $5000-\mathrm{Hz}$ off-resonance inversion pulse. Control images were collected in the absence of the inversion pulse. The relaxation of the longitudinal magnetization of the moving spins as they moved through the phantom was measured by sampling the image at different locations along the flow path. These data were compared with the predicted $T_{1}$, and $T_{1}$ sat relaxation curves.

\section{RESULTS}

\section{Simulations}

Both panels of Fig. 1 show the Bloch simulation of the longitudinal magnetization of the arterial spins as they move through the inversion plane in the presence of a magnetic field gradient and off-resonance RF radiation. Figure 1A shows the simulated inversion and relaxation process with and without exchange of protons between the free and the bound pools $\left(k_{\mathrm{a}}=0\right.$ and $35 \mathrm{~s}^{-1}$, respectively) moving at $20 \mathrm{~cm} / \mathrm{s}$. Figure $1 \mathrm{~B}$ shows the case in which magnetization transfer is present $\left(k_{\mathrm{a}}=35 \mathrm{~s}^{-1}\right)$ at different flow velocities.

In both panels, the spins start out in their relaxed state, and, as they move through the magnetic field gradient, their resonance frequency approaches that of the labeling pulse. Conversely, from their frame of reference, they experience an RF pulse that is initially far from the resonant frequency, but is closer to resonance as they approach the labeling plane. After crossing the labeling plane, the spins are inverted and relax back to their equilibrium state. During this time, their resonant frequency becomes increasingly different from the RF pulse's frequency.

In the presence of magnetization transfer, the inversion efficiency was reduced and the relaxation of the inverted spins was somewhat faster. The inversion efficiency and its reduction from magnetization transfer were both dependent on velocity. The inversion efficiency at 1, 5, 10, 15,20 and $30 \mathrm{~cm} / \mathrm{s}$ was $0.50,0.62,0.73,0.80,0.84$, and 0.90 , respectively, when magnetization transfer was considered. The loss of efficiency due to magnetization transfer was $0.21 \%, 3.65 \%, 3.50 \%, 2.85 \%, 2.24 \%$, and $1.43 \%$, respectively (only the $20 \mathrm{~cm} / \mathrm{s}$ case is shown in Fig. 1A for clarity). The relaxation rate of the inverted spins also changed as a function of velocity when magnetization transfer was included in the model.

Specifically, the difference in relaxation of the label due to magnetization transfer alone during $1 \mathrm{~s}$ at velocities of $1,5,10,15,20$, and $30 \mathrm{~cm} / \mathrm{s}$, respectively, was $-2.98 \%$, $1.89 \%, 3.36 \%, 3.45 \%, 3.38 \%$, and $3.09 \%$. The difference in relaxation, $\Delta R$, was calculated as

$$
\Delta R=100 \cdot \frac{R_{M T}-R}{R}
$$

where $R_{\mathrm{MT}}$ and $R$ are the amount of $T_{1}$ relaxation that occurred in the cases with and without consideration of magnetization transfer effects. $R$ is expressed as 
A. Effect of Magnetization Transfer

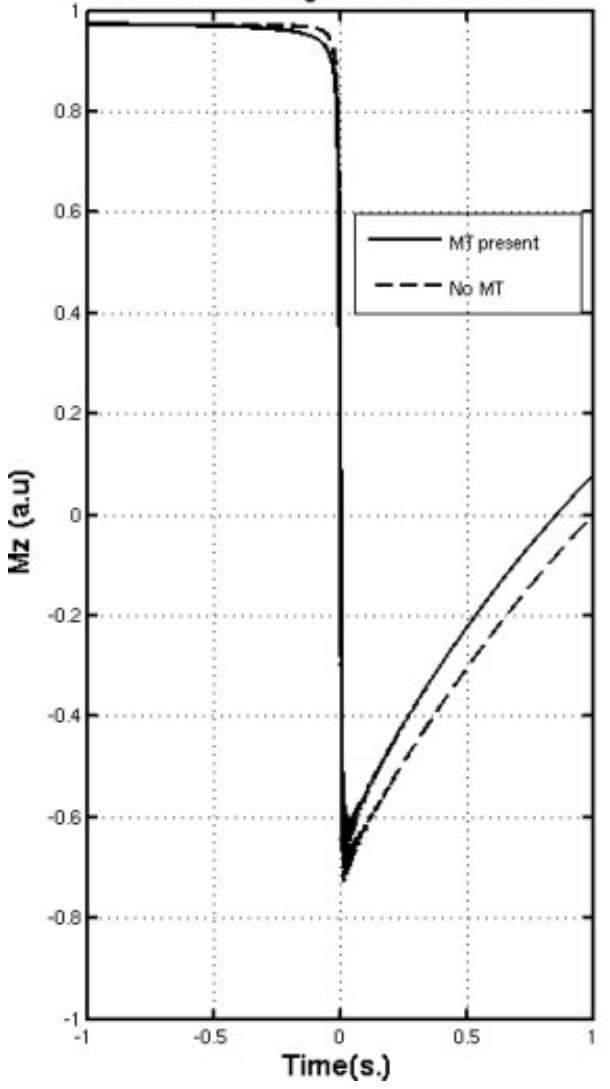

B. Effect of Velocity (MT present)

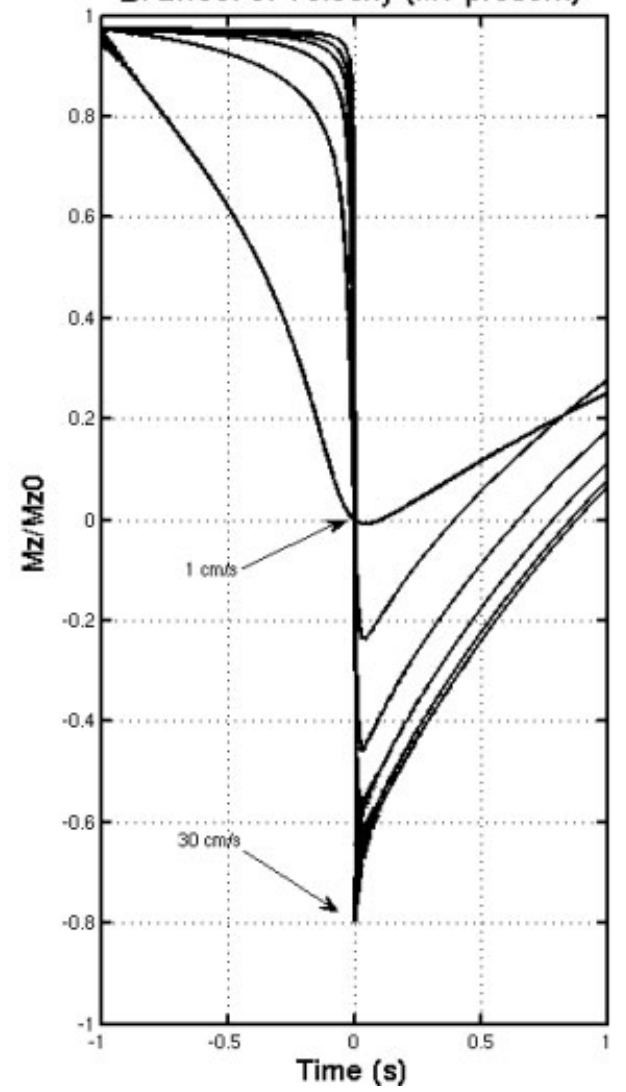

Figure 1. Simulations of adiabatic inversion of flowing spins and their subsequent relaxation in the presence of off-resonance RF. The parameters used for both simulations are shown in Table 1, unless otherwise specified. The spins cross the inversion plane at $t=0$. (A) Simulation of the effect of including magnetization transfer (MT) effects in the simulation. When the magnetization transfer effects are not considered, a greater inversion is achieved and the longitudinal relaxation rate is considerably slower. (B) Simulation of the decay of the spins at different velocities, demonstrating that slower moving spins experience a greater amount of magnetization transfer than faster ones. Note that the inversion efficiency at $1,5,10,15,20$ and $30 \mathrm{~cm} / \mathrm{s}$ was $0.50,0.62,0.73,0.80,0.84$, and 0.90 , respectively, when magnetization transfer was considered. The loss of inversion efficiency due to magnetization transfer was $0.21 \%, 3.65 \%, 3.50 \%, 2.85 \%, 2.24 \%$, and $1.43 \%$, respectively.

percentage of magnetization that was lost $1 \mathrm{~s}$ after labeling, i.e.

$$
R=100 \cdot \frac{M_{z}(1)-M_{z}(0)}{M_{z}(0)}
$$

Taken together, the reduced inversion efficiency and the change in relaxation rate, resulted in a reduction in the arterial label of $-3.96 \%, 6.66 \%, 8.91 \%, 8,51 \%, 7.92 \%$ and $6.95 \%$ after $1 \mathrm{~s}$. Note that a negative reduction means that the magnetization transfer helped to preserve the saturation state.

\section{Phantom studies: stationary phantoms}

Figure 2 shows the behavior of longitudinal magnetization in water and blood exposed to an off-resonance square pulse such as the one used for adiabatic inversion in the ASL experiment. It was noted in a preliminary experiment that approximately $1500 \mathrm{~ms}$ duration was required for equilibrium to be reached, and that there was a steady decline in the equilibrium signal as the power of the offset RF pulse was increased. The figure shows a strong dependence of saturation transfer on the frequency offset of the RF pulse, as expected. In fact, the shape of the curve is similar to other typical magnetization transfer curves $(20,21,25-27)$.

The values for $T_{1}, T_{1}$ sat, $k_{\mathrm{a}}$, and $T_{2}$ measured for blood and tap water phantoms are shown in Table 2. The forward exchange rate, $k_{\mathrm{a}}$, was $34.5 \mathrm{~s}^{-1}$ in agreement with published data (22).

\section{Phantom studies: flowing spins}

The sagittal images of flowing liquid revealed a clear laminar flow profile (Fig. 3). The velocity of the flowing 
Frequency Dependence of Magnetization Transfer

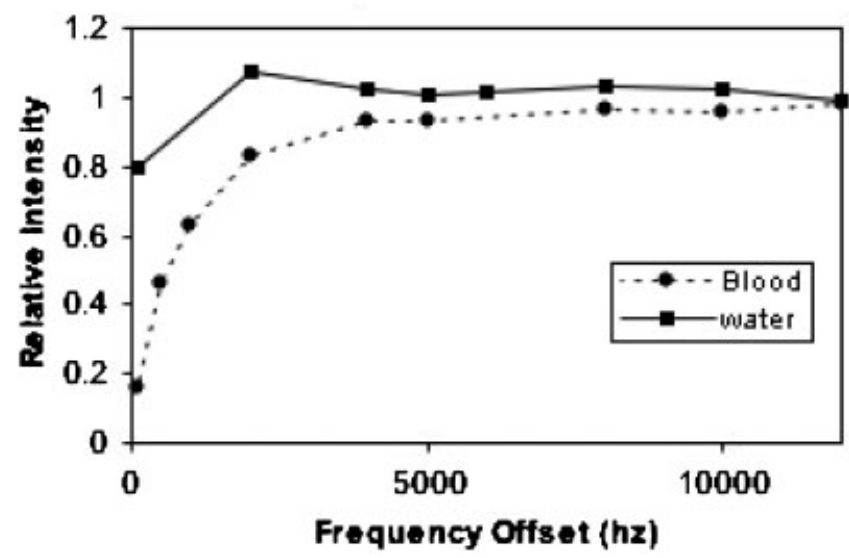

Figure 2. Magnetization transfer spectrum of water and blood. The curve shows the typical frequency dependence observed in tissues and blood.

Table 2. Measured constants

\begin{tabular}{lcccc}
\hline & $T_{1}(\mathrm{~ms})$ & $T_{2}(\mathrm{~ms})$ & $T_{1}$ sat $(\mathrm{ms})$ & $k_{\mathrm{a}}\left(\mathrm{s}^{-1}\right)$ \\
\hline Water & 2500 & 365 & - & - \\
Blood & 1810 & 62 & 1357 & 34.5 \\
\hline
\end{tabular}

spins measured from the sagittal images ranged from 1 to $20 \mathrm{~cm} / \mathrm{s}$. The degree of inversion as a function of RF power was measured from cross-sectional images in both blood and water at different flow velocities. Figure 4A shows a plot of the maximum inversion achievable in both water and blood as a function of flow velocity. Nearly $100 \%$ inversion was quickly achieved in water, while the degree of inversion in blood correlated strongly and positively with velocity. The maximum degree of inversion achieved in blood was considerably

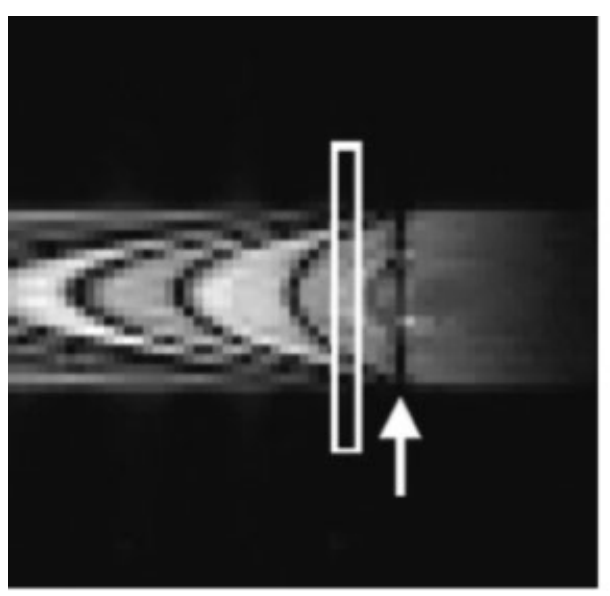

A

Maximum Inversion Dependence on Flow Velocity

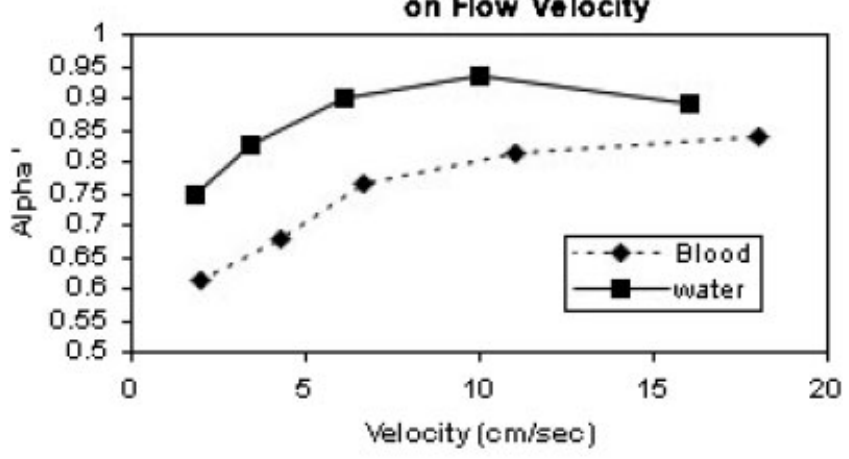

B

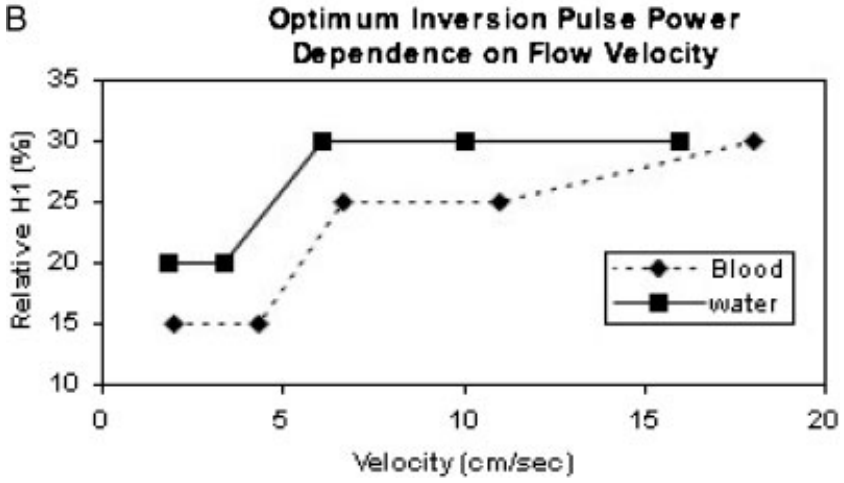

Figure 4. (A) Maximum inversion achieved as a function of velocity. At low flow velocities, the maximum inversion observed is lower than at higher flow velocities because of saturation due to magnetization transfer. (B) Plot of power required for optimum inversion efficiency. Inversion efficiency (alpha) was measured using eqn (2). The optimum inversion power depends not only upon the velocity, but also upon the contribution of magnetization transfer, as demonstrated by the difference between the water and blood phantoms.

lower in the slow flow cases, and it was always lower than in blood. Note that the inversion efficiency is always less in blood than in water because of the difference in relaxation parameters, in addition to the magnetization

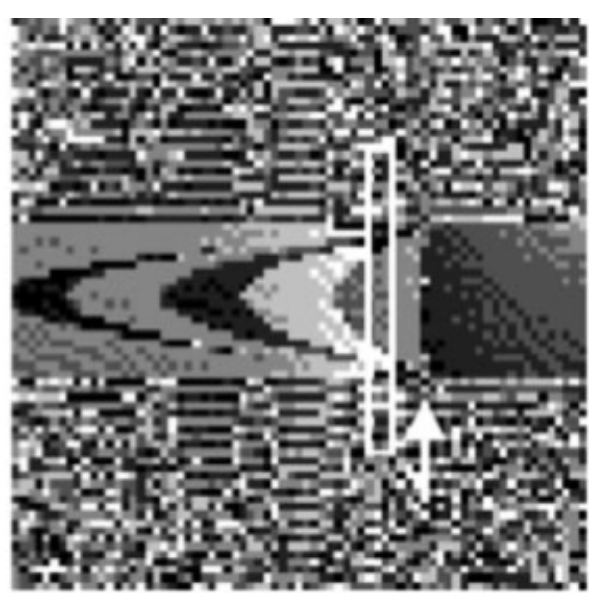

Figure 3. Magnitude (left) and phase (right) images showing laminar flow in phantom. Inversion pulses lasted $200 \mathrm{~ms}$, while image acquisition parameters were $T R=300$, $T E=21 \mathrm{~ms}$. The rectangle identifies the plane used for measurement of inversion efficiency as a function of velocity. Arrows indicate plane of application of the adiabatic inversion pulse. Multiple banding patterns in both the magnitude and phase images arise from the alternating control and labeling pulses applied before imaging. 
A

Measured T1 Decay During Transit

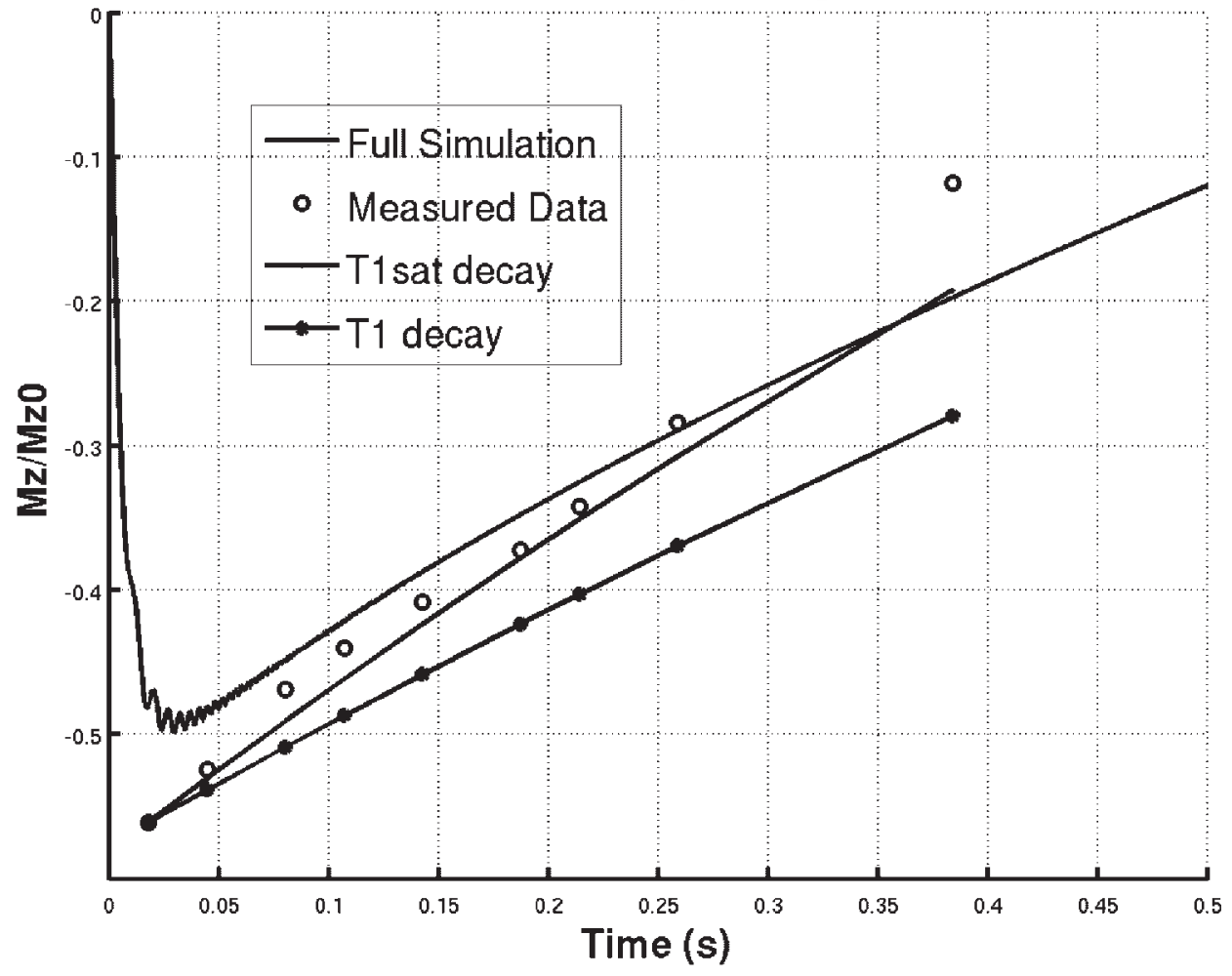

B

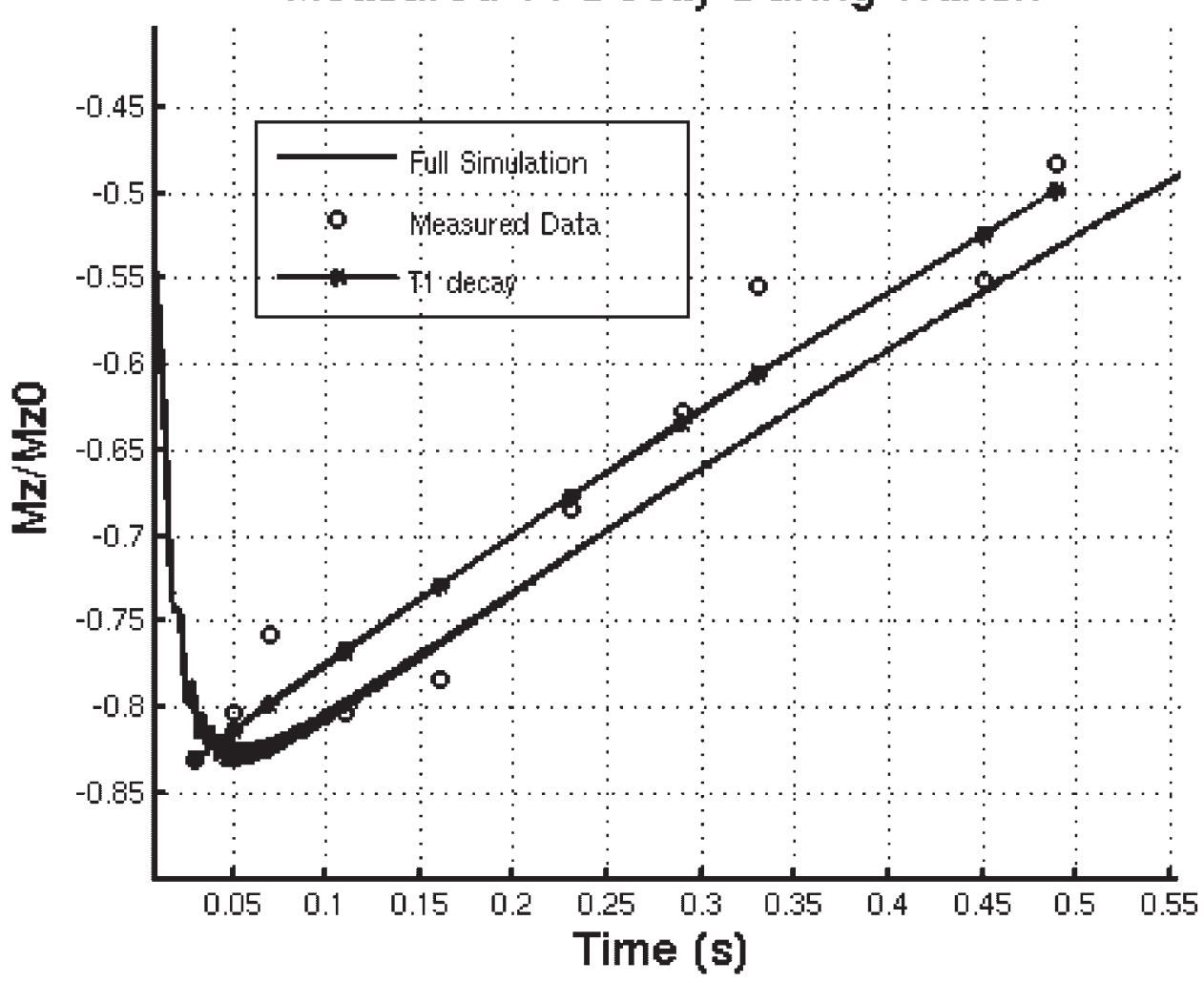

Figure 5. Relaxation of inverted spins in blood (A) and water (B) as they move away from the inversion plane. The data are compared with $T_{1}$ and $T_{1}$ sat relaxation, as well as the model presented here. The relaxation rate appears to match the $T_{1}$ sat relaxation curve more closely than the $T_{1}$ relaxation curve. 
transfer effects. A plot of the RF pulse power required to achieve the maximum attainable degree of inversion versus flow velocity is shown in Fig. 4B. The $H_{1}$ field required to achieve inversion increased with the square of the velocity, consistently with the adiabatic condition (eqn 1). In other words, as flow velocity increased, the power required for maximum inversion increased as well.

In the ASL experiment, arterial water spins continue to be exposed to off-resonance radiation after they are inverted at the inversion plane. Analogously, Fig. 5A shows the relaxation of inverted spins in blood as they move away from the inversion plane in the presence of off-resonance RF radiation. In the same plot, are the calculated blood $T_{1}$ and $T_{1}$ sat relaxation curves, along with the decay predicted by the full model accounting for MT effects (eqns 7 and 8 with the values in Tables 1 and 2). Interestingly, blood spins appear to relax faster than $T_{1}$ relaxation would predict. The mean squared error (MSE), relative to the empirical data, was 0.046 for the $T_{1}$ decay prediction, 0.0075 for the $T_{1}$ sat prediction, and 0.017 for the full simulation prediction. Figure 5B illustrates the relaxation of inverted spins in water under similar conditions to those of blood in Fig. 5A. In water, the relaxation of inverted spins closely followed $T_{1}$ relaxation. The MSE for the simple $T_{1}$ decay prediction was 0.0099 and 0.0181 for the full simulation.

\section{DISCUSSION}

These data point out two issues that must be considered in order to correctly predict the behavior of the inverted spins in the ASL measurement. First, the velocity dependence of the adiabatic condition introduces variability in the degree of inversion obtained by a given RF pulse with velocity fluctuations. As indicated by others $(16,19,17,18)$, appropriate choice of gradient magnitude and $H_{1}$ strength will minimize this effect, although at the expense of greater specific absorption rate.

Second, the degree of magnetization transfer which occurs in the blood may be more significant than originally proposed. Both the model and phantom data illustrate a loss of inversion from exposure to offresonance $\mathrm{RF}$ radiation during transit from the inversion plane to the slice of interest. The inversion loss is more dramatic at low velocities than at high velocities, which is in agreement with the study by Dousset et at. (10). On the basis of the magnitude of the magnetization transfer inversion loss observed at the lower velocities, the contribution of this effect in vivo may be quite significant. For example, in the case of $20 \mathrm{~cm} / \mathrm{s}$ flow velocity (when tagging just below the circle of Willis), one loses approximately $42 \%$ of the label in $1 \mathrm{~s}$ of transit time (i.e. our simulations predict that the inversion efficiency, $\alpha$, at the time the label reaches the tissue is reduced by $42 \%$ ). Our calculations show that approximately an additional $8 \%$ is lost due to the lower efficiency of inversion and the change in relaxation rate (see our simulations above). In other words, this effect may be quite significant relative to the proposed $T_{1}$ decay during spin transit periods (19). At the same time, when the efficiency of the label was around 0.5 (saturation) as in the $1 \mathrm{~cm} / \mathrm{s}$ case, the exchange between free and bound pools consisted of saturated spins only, and the relaxation rate change is less noticeable. Thus, there is a trade off between the loss of labeling efficiency and the change in effective $T_{1}\left(T_{1}\right.$ sat) due to magnetization transfer. Labeling is less efficient at slower velocity, such that the label consists of saturation rather than inversion in the slowest cases. Subsequently, the off-resonance RF radiation helps to preserve the saturation state as the spins spend more time near the resonance plane. At faster velocity, greater inversion efficiency is achieved, but the off-resonance RF pulses cause the well-known shortening of $T_{1}$. At the same time, as velocity increases, the spins can get away from the resonance plane faster, and the amount of magnetization transfer and concomitant $T_{1}$ shortening are reduced.

According to Fig. 5A,B, our Bloch simulations yielded larger MSE values than simple $T_{1}$ sat decay calculations based on the efficiency measured immediately after the spins cross the labeling plane, but smaller than the $T_{1}$ decay predictions. The laminar flow profile of the flow through the phantom, along with uncertainty in the constants, contributed to capture the features the error in the Bloch simulations. Despite the increased error, the Bloch simulations do capture the features of the inversion. In practical terms, however, one must calculate the decay of the label in order to quantify perfusion from the ASL signal. The most accurate method to calculate relaxation of the label appears to be the use of simple $T_{1}$ sat decay, rather than full Bloch simulations. Both the $T_{1}$ sat and full Bloch simulations are more accurate than the usual $T_{1}$ decay of blood.

We made some assumptions in the formulation of our model. In our simulations, we have assumed arterial blood flow to be uniform, plug flow between the inversion plane and the slice of interest. Arterial flow is pulsatile and turbulent. At the same time, the tortuosity and variations in the angle between the carotid arteries and the $z$-axis contribute to variations in the $z$-component of the arterial velocity. The effects of the dispersion of arterial velocities were examined by Hrabe \& Lewis (28), who characterized the dispersion as a Gaussian distribution of transit times. These variations contribute to the residency time of the spins in the $H_{1}$ field. We do not have measurements for $T_{1}, T_{2}$ of bound protons in blood, but we have used an approximation of their value in the computations, in order to illustrate the behavior of the signal with various velocities and magnetization transfer. The value for the bound $T_{2}$ is that reported in [22] at $3 \mathrm{~T}$, and the bound $T_{1}$ value is merely an educated guess, but this parameter has a negligible effect on the simulations. Thus, our numerical simulations contain a certain degree of uncertainty and must be viewed as an aid to illustrate 
the point, rather than a quantitative measure of the degree of inversion during the transit time.

It should also be noted that the experiments carried out in this work were performed on venous human blood at room temperature for ease of implementation, whereas the conditions in ASL experiments are those of body temperature and arterial blood. Hence, in vivo experiments have shorter $T_{1}$ and longer $T_{2}$ relative to our experimental conditions. We anticipate that the magnetization transfer effect (the main thrust of this work) of our experimental setup should remain very similar to in vivo experiments.

The effective degree of inversion, $\alpha^{\prime}$, accounts for the $T_{1}$ decay of the inversion label during transit time from the inversion plane to the image slice, but it does not account for the effects of magnetization transfer. In the case of inverted stationary spins relaxing in the presence of off-resonance RF, one could approximate $\alpha^{\prime}$ by using the $T_{1}$ sat relaxation rate instead. Ordidge et al. (25) measured $T_{1}$ sat in tissue in the presence of varying frequency offset RF pulses. Their data suggest that $T_{1}$ sat gets shorter with frequency offset, and it approaches $T_{1}$ as the offset increases. In the case of flowing blood, the frequency offset of the RF experienced by the arterial spins varies as they travel away from the inversion plane, suggesting relaxation with variable $T_{1}$ sat. Figure 5 suggests that the spins relax with shorter $T_{1}$ sat early on their way to the imaging slice, but as they get further away, they relax with $T_{1}$ rate. In single-coil applications, it is advantageous to use a large frequency offset so that the relaxation rate approaches the $T_{1}$ sooner, hence preserving the inversion label longer. Because of the relatively short distance between the inversion plane and the image plane, using $T_{1}$ sat for the computation of $\alpha^{\prime}$ instead of $T_{1}$ yields a better approximation to the real $\alpha^{\prime}$.

Our data suggest that macromolecular spins in arterial blood are also saturated by the inversion pulse, which accounts for loss of the inversion tag by saturation transfer. Our data as well as the data collected by Dousset et al. (10) suggest that saturation transfer in blood increases at lower flow velocities. The implication is that this effect should be more pronounced in human ASL studies than in rat studies, where the inversion plane is not placed at the carotid arteries, but a few centimeters before the slice of interest, where flow occurs through smaller vessels with slower flow velocities. In continuous ASL studies, the labeled blood is exposed to the off-resonance $\mathrm{RF}$ for long periods of time (of the order of $4 \mathrm{~s}$ ) while it builds up a steady-state level in the tissue. The blood is also decelerating while it travels to the tissue, which further exacerbates the magnetization transfer effect. Although our simulations and phantom experiments are a simplification of the problem, we estimate that roughly $5-10 \%$ of the label is lost due to magnetization transfer effects in typical ASL human experiments. Using a separate labeling coil over the carotid and vertebral arteries at the neck allows labeling of the arterial blood at higher velocity, improving the inversion efficiency at the cost of increased transit time. In addition, using a separate labeling coil reduces the amount of off-resonance RF radiation experienced by the flowing spins and therefore the loss of efficiency.

\section{Acknowledgements}

We thank Drs Stephan Roppele and Greg Stanisz for their valuable comments. This work is supported by the National Institute of Biomedical Imaging and Bioengineering (1 R01 EB004346-01A1).

\section{REFERENCES}

1. Calamante F, Williams SR, van Bruggen N, Kwong KK, Turner R. A model for quantification of perfusion in pulsed labelling techniques. NMR Biomed. 1996; 9: 79-83.

2. Kim SG. Quantification of relative cerebral blood flow change by flow-sensitive alternating inversion recovery (FAIR) technique: application to functional mapping. Magn. Reson. Med. 1995; 34: 293-301.

3. Kwong KK, Cheslar DA, Weisskoff RM, Donahue KM, Davis TL, Ostergaard L. MR perfusion studies with T1-weighted echo planar imaging. Magn. Reson. Med. 1995; 34: 878-887.

4. Wong EC, Buxton RB, Frank LR. Quantitative imaging of perfusion using a single subtraction (QUIPSS and QUIPSS II). Magn. Reson. Med. 1998; 39: 702-708.

5. Alsop DC, Detre JA. Reduced transit-time sensitivity in noninvasive magnetic resonance imaging of human cerebral blood flow. J. Cereb. Blood Flow Metab. 1996; 16: 1236-1249.

6. Roberts DA, Detre JA, Bolinger L, Insko EK, Leigh JSJ. Quantitative magnetic resonance imaging of human brain perfusion at 1.5 T using steady-state inversion of arterial water. Proc. Natl. Acad. Sci. USA. 1994; 91: 33-37.

7. Williams DS, Detre JA, Leigh JS, Koretsky AP. Magnetic resonance imaging of perfusion using spin inversion of arterial water. Proc. Natl. Acad. Sci. USA. 1992; 89: 212-216.

8. Zhang W, Williams DS, Koretsky AP. Measurement of rat brain perfusion by NMR using spin labeling of arterial water: in vivo determination of the degree of spin labeling. Magn. Reson. Med. 1993; 29: 416-421.

9. Lee HK, Nalcioglu O, Moran PR. Spatially resolved flow velocity measurements and projection angiography by adiabatic passage. Magn. Reson. Imaging. 1991; 9: 115-127.

10. Dousset V, Degreze P, Mieze S, Sesay M, Basse-Cathalinat B, Caille JM. Magnetization transfer on in vitro circulating blood: implications for time-of-flight MR angiography. J. Magn. Reson. Imaging. 1995; 5: 786-788.

11. Pike GB, Hu BS, Glover GH, Enzmann DR. Magnetization transfer time-of-flight magnetic resonance angiography. Magn. Reson. Med. 1992; 25: 372-379.

12. Branch CA, Hernandez L, Lewis DP. Degradation of efficiency of steady state arterial inversion due to magnetization transfer in the blood: further evidence. Proceedings International Society for Magnetic Resonance in Medicine 2000; 164.

13. Hernandez L, Branch CA. Theoretical effects of magnetization transfer on inversion efficiency in steady state arterial inversion for measurement of cerebral perfusion. Proceedsings ISMRM 1998; 1218.

14. Garcia DM, Duhammel G, Alsop DC. Slow magnetization transfer dominates inefficiency of suppressed arterial spin labeling. International Society for Magnetic Resonance in Medicine 2005; 13: 1151.

15. Roberts DA, Bolinger L, Detre JA, Insko EK, Bergey P, Leigh JSJ. Continuous inversion angiography. Magn. Reson. Med. 1993; 29: 631-636. 
16. Marro KI, Hayes CE, Kushmerick MJ. A model of the inversion process in an arterial inversion experiment. NMR Biomed. 1997; 10: $324-332$.

17. Gach HM, Kam AW, Reid ED, Talagala SL. Quantitative analysis of adiabatic fast passage for steady laminar and turbulent flows. Magn. Reson. Med. 2002; 47: 709-719.

18. Utting JF, Thomas DL, Gadian DG, Ordidge RJ. Velocity-driven adiabatic fast passage for arterial spin labeling: results from a computer model. Magn. Reson. Med. 2003; 49: 398-401.

19. Maccotta L, Detre JA, Alsop DC. The efficiency of adiabatic inversion for perfusion imaging by arterial spin labeling. NMR Biomed. 1997; 10: 216-221.

20. Henkelman RM, Huang X, Xiang QS, Stanisz GJ, Swanson SD, Bronskill MJ. Quantitative interpretation of magnetization transfer. Magn. Reson. Med. 1993; 29: 759-766.

21. Morrison C, Henkelman RM. A model for magnetization transfer in tissues. Magn. Reson. Med. 1995; 33: 475-482.

22. Stanisz GJ, Odrobina EE, Pun J, Escaravage M, Graham SJ, Bronskill MJ, Henkelman RM. T1, T2 relaxation and magnetization transfer in tissue at 3T. Magn. Reson. Med. 2005; 54: 507-512.
23. Branch CA, Hernandez L, Yongbi M, Huang NC, Helpern JA. Rapid and continuous monitoring of cerebral perfusion by magnetic resonance line scan assessment with arterial spin tagging. NMR Biomed. 1999; 12: 15-25.

24. Gudbjartsson H, Maier SE, Mulkern RV, Morocz IA, Patz S, Jolesz FA. Line scan diffusion imaging. Magn. Reson. Med. 1996; 36: 509-519.

25. Ordidge RJ, Helpern JA, Knight RA, Qing ZX, Welch KM. Investigation of cerebral ischemia using magnetization transfer contrast (MTC) MR imaging. Magn. Reson. Imaging 1991; 9: 895-902.

26. Ordidge RJ, Knight RA, Helpern JA. Magnetization transfer contrast (MTC) in flash MR imaging. Magn. Reson. Imaging 1991; 9: 889-893.

27. Wolff SD, Balaban RS. Magnetization transfer contrast (MTC) and tissue water proton relaxation in vivo. Magn. Reson. Med. 1989; 10: $135-144$.

28. Hrabe J, Lewis DP. Two analytical solutions for a model of pulsed arterial spin labeling with randomized blood arrival times. J. Magn. Reson. 2004; 167: 49-55. 